\title{
ELECTROCHEMICAL REDUCTION OF THYMINE IN DIMETHYL SULFOXIDE AUTOPROTONATION OF THE RADICAL ANION AND REDUCED FREE RADICAL
}

\author{
TIMOTHY E. CUMMINGS
}

University of Miami, Coral Gables, FL 33124 (U.S.4.)

PHILIP J. ELVING

University of Michigan, Ann Arbor, MI 48109 (U.S.A.)

(Received 17th August 1978; in revised form 3rd January 1979)

\section{ABSTRACT}

Thymine is reduced in dimethyl sulfoxide $\left(E_{1 / 2}\right.$ of $\left.-2.4 \mathrm{~V}\right)$; the decrease in apparent faradaic $n$ from one at infinite dilution to two-thirds at concentrations above $2 \mathrm{~m} M$ is due to proton transfer by thymine to the initially formed radical anion and the anion formed on reduction of the resulting free radical, which is more easily reduced than the parent compound. The proton transfer, which is more rapid than the radical anion dimerization, deactivates part of the thymine, forming the more difficuitly reducible conjugate base. The latter forms insoluble mercury salts, producing up to three oxidation waves $\left(E_{1 / 2}\right.$ between -0.08 and $-0.3 \mathrm{~V}$ ). The free radical reduction, which is not observed for uracil or 2-hydroxypyrimidine, occurs due to steric hindrance by the 5 -methyl group toward free radical dimerization, thereby increasing the half-life of the free radical. The effect of added base on the electrochemical behavior is described.

\section{INTRODUCTION}

Recently, the electrochemical reductions in dimethyl sulfoxide (DMSO) of 2-hydroxypyrimidine (2-HP) [1] and uracil (2,4-dihydroxypyrimidine) [2] were described. The electrochemical reduction of thymine (5-methyl-2,4-dihydroxypyrimidine), in addition to the intrinsic interest in thymine as a constituent of DNA, may aid in clarifying the specific patterns of 2-HP and uracil reduction, since the 5-methyl group of thymine is close to the postulated C(4) site of addied electron residence and dimerization in both previously studied compounds $[1,2]$.

In aqueous and non-aqueous media, the keto-enol equilibrium for hydroxypyrimidines very strongly favors the keto form [3]:<smiles>C=C</smiles> 
In non-aqueous media (DMSO), thymine, similarly to 2-HP and uracil, is initially reduced in a one-electron (1e) step to the radical anion, which reacts with unreduced thymine to produce the neutral free radical and thymine's conjugate. base; radical anion dimerization is slower than the competing proton transfer. The reduction mechanism of thymine differs from those for uracil and 2-HP in that, for the latter, the free radical rapidly dimerizes, whereas the thymine free radical is quantitatively reduced to an anion, which also abstracts a proton from thymine to form dihydrothymine and additional conjugate base.

The general nature of the proton transfer, or father-son reaction, and its influence on observed polarographic and voltammetric current and potential response have been discussed $[1,2,4]$.

\section{EXPERIMENTAL}

\section{Chemicals}

Thymine was obtained from National Biochemicals; aqueous 20\% tetraethylammonium hydroxide (TEAH) solution from Aldrich. A $3 \mathrm{M} \mathrm{HClO}{ }_{4}$ solution in DMSO was prepared from aqueous $1: 1$ diluted reagent grade perchloric acid (G. Frederick Smith) (dilution of $\mathrm{HClO}_{4}$ directly with DMSO results in exothermic oxidation of the DMSO). Reagent grade tetra-n-butylammonium perchlorate (TBAP) (G. Frederick Smith) was vacuum dried at $60^{\circ} \mathrm{C}$ for $48 \mathrm{~h}$. DMSO (Fisher certified ACS; Mallinckrodt analytical reagent; "Baker Analyzed" reagent) was purified by fractional freezing [5]; the cooling bath was $\mathrm{H}_{2} \mathrm{O}$ at $12^{\circ} \mathrm{C}$ to $14^{\circ} \mathrm{C}$. Mercury for electrodes was distilled.

\section{Apparatiss}

A Frinceton Applied Research PAR 174 potentiostat was used with a jacketed three-compartment cell thermostatted al $25^{\circ} \mathrm{C}$. For rapid cyclic voltammetry, a fast response potentiostat, a single-chamber cell with Luggin capillary, and a faradaic cage were used [6]. The hanging mercury drop electrode was a Metrohm E 410 microfeeder. A Wavetek Model 112 function generator supplied triangular wave forms. Data were acquired on Hewlett-Packard 7005B or Houston $2000 \mathrm{XY}$ recorders, and a Tektronix 502 oscilloscope or a $5103 \mathrm{~N}$ with $5 \mathrm{~A} 15 \mathrm{~N}$ and $5 \mathrm{A18N}$ plug-ins, using a C-5A or C-12 camera. Potentials, monitored with a Hewlett-Packard 3430A or 3440A digital voltmeter, were measured against a modified aqueous saturated calomel electrode (SCE) by the following system: SCE/saturated $\mathrm{KCl}$ in water-methylcellulose bridge with asbestos fiber. A Unimetrics $101010-\mu \mathrm{l}$ syringe was used for addition of acid and base solutions.

\section{Procedures}

Solutions (0.1 $M$ TBAP as background electrolyte) were deoxygenated by purging with purified $\mathrm{N}_{2}$ for ca. $30 \mathrm{~min}$; an $\mathrm{N}_{2}$ atmosphere was maintained in the cell throughout an experiment. D.c. polarographic data were obtained at a controlled 2.0-s drop-time, using a capillary with open-circuit mercury flow-rate 
of $0.575 \mathrm{mg} \mathrm{s}^{-1}$. A new Hg drop (effective area $=0.0153 \mathrm{~cm}^{2}$ [7]) was used for each cyclic voltammogram.

For controlled potential electrolysis, a $7-\mathrm{cm}^{2}$ mercury pool was used. Separate electrolyses were performed on $25.0 \mathrm{ml}$ of background and thymine solutions; $\mathrm{N}_{2}$ was used to continuously stir the solution. Current-time profiles were recorded.

Experiments showed no effect on electrochemical behavior from the amounts of water involved in adding $\mathrm{TEAH}$ or $\mathrm{HClO}_{4}$.

Roman numbers are used to identify waves and peaks with appended $c$ or $a$ to indicate cathodic or anodic processes, respectively.

\section{Cyclic voltammetric peak charge calculation}

Because of the apparent filming nature of some faradaic peaks, the number of coulombs, $Q$, passed in faradaic peaks was determined. The peak area was defined by extrapolation of the charging current to form a baseline; integration for peak IIC was performed over the potential span from the peak foot to the potential at which the current returned to the baseline; integration of the total area for peaks Ia, IIa and IIIa was performed from the peak Ia foot to the switching potential for the positive scan and from the switching potential to the point at which the current crossed the baseline for the negative scan. The integrated area (measured by a Gelman Model 39231 planimeter) was multiplied by the read-out device $E$ - and $i$-axis sensitivities and divided by the scanrate to yield the charge passed in $\mu \mathrm{C}$.

\section{RESULTS AND DISCUSSION}

\section{D.c. polarography}

The concentration-dependence of the thymine diffusion current constant $\left(I_{\mathrm{d}}=\bar{i}_{1} / \mathrm{cm}^{2 / 3} i^{1 / 6}\right)$ is shown in Fig. 1 ; extrapolation at low c to infinite dilution yields an $I_{\mathrm{d}}$ of $1.3 \mu \mathrm{A} \mathrm{s}^{1 / 2} \mathrm{mM}^{-1} \mathrm{mg}^{-2 / 3}$, which corresponds to a diffusion coefficient, $D$, of $4.6 \times 10^{-6} \mathrm{~cm}^{2} \mathrm{~s}^{-1}$. Use of the Matsuda equation [8], which considers shielding effects and best describes the $i_{\mathrm{d}}-t$ behavior at controlled drop-times [7], gives a $D$ of $4.0 \times 10^{-6}$. The data at $c$ exceeding $1.7 \mathrm{mM}$ yield an $I_{d}$ of 0.77 .

$E_{1 / 2}$ is $-2.41 \mathrm{~V}$ at $0.06 \mathrm{~m} M,-2.39 \mathrm{~V}$ at $0.2 \mathrm{~m} M$, and $-2.36 \mathrm{~V}$ above $1 \mathrm{~m} M$. Although this shift is near experimental uncertainty $(0.01 \mathrm{~V})$, it parallels the concentration-dependence of $I_{\mathrm{d}}$ and may, therefore, be real. 'The $E_{1 / 2}-c$ variation closely parallels that for uracil; $E_{1 / 2}$ for thymine is ca. $0.07 \mathrm{~V}$ negative of that for uracil below $1 \mathrm{~m} M$ and ca. $0.05 \mathrm{~V}$ above $1 \mathrm{~m} M$.

Thymine and uracil [2] have an infinite dilution $I_{\mathrm{d}}$ of 1.3, which, presumably, represents a $1 e$ reduction; the $I_{\mathrm{d}}$ of 0.77 above $1 \mathrm{~m} M$, which is $15 \%$ larger than that for uracil, represents an $n$ of 0.59 . Thus, at high $c$, either $31 \%$ of the radical anion formed on le reduction dimerizes with $69 \%$ being protonated by unreduced thymine or part of the free radical formed on protonation is reduced in an e.c.e. reaction. Dimerization of a significant fraction of radical anion implies a reversal in the relative protonation and dimerization rates as compared 


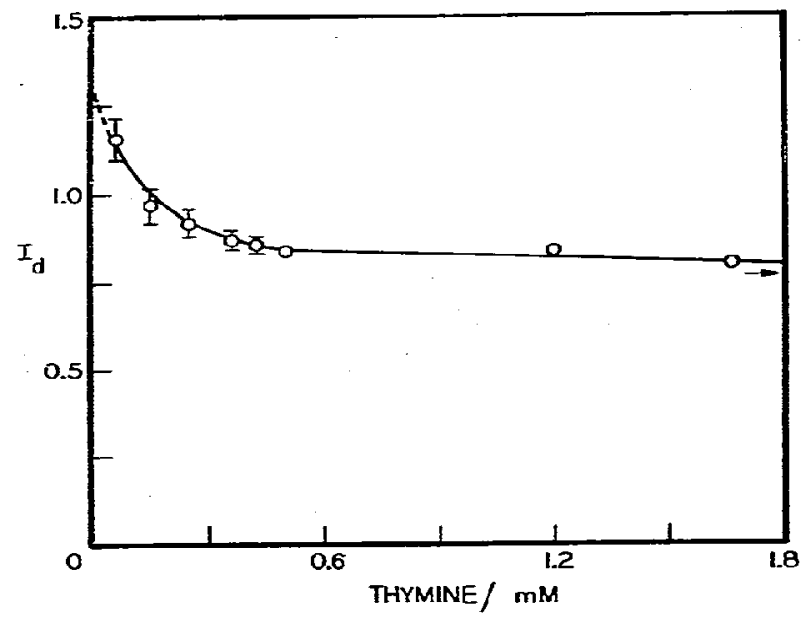

Fig. 1. Concentration-dependence of the d.c. polarographic diffusion current constant $\left(I_{\mathrm{d}}\right)$ for thymine, based on mean currents. The horizontal arrow corresponds to $I_{\mathrm{d}}$ at 2.0 and $2.5 \mathrm{~m} M$. Uncertainties are one standard deviation.

to uracil, for which virtually $100 \%$ of the radical anion is protonated. An e.c.e. mechanism requires that the free radical dimerization be considerably slower than that for uracil, which consumes $100 \%$ of the free radical.

\section{Cyclic voltammetry}

The cyclic voltammetric behavior of thymine (Figs. 2 and 3 ) is similar to those of 2-HP [1] and uracil [2]. Reversal of the potential scan negative of the thymine reduction produces anodic peaks positive of $-0.3 \mathrm{~V}$; when the scan is again reversed, a cathodic peak (IIc) is seen at ca, $-0.3 \mathrm{~V}$, which has the characteristic shape of film stripping. At $v$ of 600 to $1200 \mathrm{~V} \mathrm{~s}^{-1}$, a minute anodic peak, possibly due to radical anion or free radical oxidation, occasionally appears about $0.2 \mathrm{~V}$ positive of the thymine reduction peak.

At slow $v$ and thymine anion concentrations above $0.8 \mathrm{mM}$, three anodic peaks appear. Two unresolved or partially resolved peaks constitute IIc (Fig. 3). The film behavior suggests significant differences in ease of formation and reduction of different salt layers (cf. Nature of thymine-mercury film).

At 0.06 to $0.35 \mathrm{~V} \mathrm{~s}^{-1}, E_{\mathrm{p}}$ shifts linearly with $\log v\left(-0.06 \mathrm{~V}\right.$ decade $\left.{ }^{-1}\right)$; at $0.12 \mathrm{~V} \mathrm{~s}^{-1}, E_{\mathrm{p}}$ is $-2.44 \mathrm{~V}$, independent of concentration (0.43 to $2.5 \mathrm{mM}$ ). Background impurity problems at potentials negative of $-2.5 \mathrm{~V}$ caused uncertainties in $i_{\mathrm{p}}$ evaluation at higher $v$.

The peak current function, $i_{\mathrm{p}} / A c v^{1 / 2}$, is 281 for $0.43 \mathrm{~m} M$ thymine at $v$ above $0.1 \mathrm{~V} \mathrm{~s}^{-1}$ and 248 for $2 \mathrm{mM}$ at $0.35 \mathrm{~V} \mathrm{~s}^{-1}$; the predicted faradaic $n$ is 0.67 at $0.43 \mathrm{~m} M$ and 0.59 at $2.0 \mathrm{mM}$, based on theoretical $i_{\mathrm{p}} / A c v^{1 / 2}$ of 422 for an irreversible $1 e$ transfer with a transfer coefficient, $\alpha$, of 0.5 and $D$ of $4 \times 10^{-6}$. These $n$ values predict $I_{\mathrm{d}}$ within \pm 0.01 of those observed. Thus, the $i_{\mathrm{p}} / A c v^{1 / 2}$ concentration-dependence parallels that of $I_{\mathrm{d}}$. 

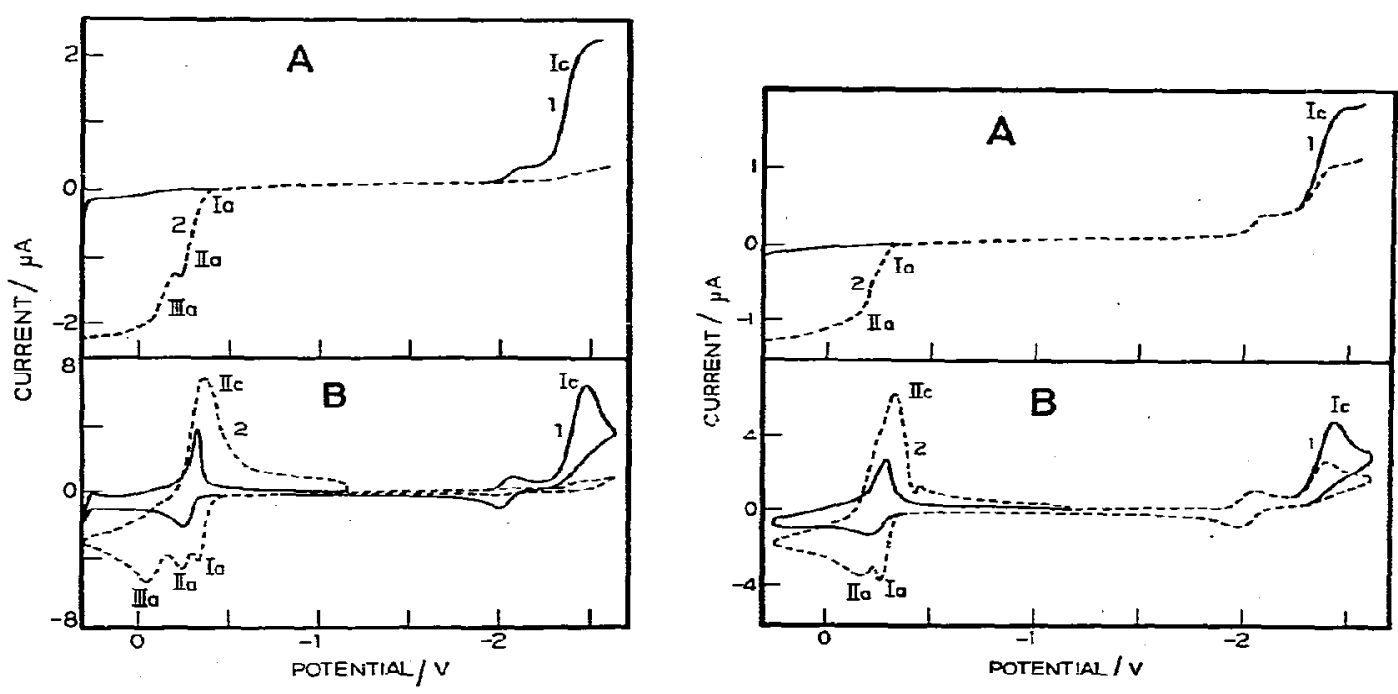

Fig. 2. D.c. polarographic and cyclic voltammetric behavior of a $2.50 \mathrm{~m} M$ thymine solution. Curves 1 (solid lines) are before controlled potential electrolysis; curves 2 (dashed lines) are after electrolysis; where curves 1 and 2 coincide, only the dashed line is shown. (A) D.c. polarograms with $2 \mathrm{~s}$ drop-time and $0.58 \mathrm{mg} \mathrm{s}^{-1}$ mercury flow-rate; (B) cyclic voltammograms with $0.06 \mathrm{~V} \mathrm{~s}^{-1}$ scan-rate and $0.0153 \mathrm{~cm}^{2}$ electrode area. The unlabelled wave and peaks between -2.0 and $-2.1 \mathrm{~V}$ are due to a solvent impurity.

Fig. 3. Effect of added base on the d.c. polarographic and cyclic voltammetric behavior of $1.67 \mathrm{~m} M$ thymine solution. Curves 1 (solid lines) are without added base; curves 2 (dashed lines) are with $0.92 \mathrm{mM}$ TEAH present; where curves 1 and 2 coincide, only the dashed line is shown. (A) D.c. polarograms with $2 \mathrm{~s}$ drop-time and $0.58 \mathrm{mg} \mathrm{s}$-1 mercury flow-rate; (B) cyclic voltammograms with $0.06 \mathrm{~V} \mathrm{~s}^{-1}$ scan-rate and $0.0153 \mathrm{~cm}^{2}$ electrode area. The unlabelled wave and peaks between -2.0 and $-2.1 \mathrm{~V}$ are due to a solvent impurity.

\section{Effect of added acid}

The only available protonation sites on thymine are the acidic exocyclic oxygens. Since added acid could only serve as a proton donor, replacing thymine in the protonation steps following reduction, the effect of added acid was not investigated.

\section{Effect of added base}

Addition of base (TEAH) at $1: 1$ ratio for $1.67 \mathrm{mM}$ thymine decreases wave Ic and produces waves Ia to IIIa $\left(E_{1 / 2}=-0.3,-0.23,-0.08\right.$; Fig. 3 ; Table 1$)$; polarographic and voltammetric behavior of the electrolyzed soiution (Fig. 2) are equivalent to that of $1.67 \mathrm{mM}$ thymine with $1: 1$ added TEAH (cf. c.p.e. discussion). $E_{1 / 2}$ shifts slightly negatively with increasing added base, e.g., -0.03 and $-0.04 \mathrm{~V}$ for Ia and IIa, respectively, for a two-fold increase; $E_{1 / 2}$ for IIIa is independent of anion concentration. $E_{p}$ shifts parallel those of $E_{1 / 2}$ - At the potential wave Ia reaches an $i_{1}$ density of ca. $45 \mu \mathrm{A} \mathrm{cm}^{-2}$ without a discernable plateau, Ila begins to appear; distinction between the two waves is evident from 
TABLE :

Variation of the DME waveheights for $1.67 \mathrm{mM}$ thymine in DMSO on addition of tetraethylammonium hydroxide

\begin{tabular}{|c|c|c|c|c|}
\hline \multirow[b]{2}{*}{$\begin{array}{l}\text { [TEAH] } \\
\mathrm{m} M\end{array}$} & \multicolumn{3}{|c|}{$i_{1} / \mu \mathrm{A}$ for wave } & \multirow[b]{2}{*}{$I_{\mathrm{d}}{ }^{a}$} \\
\hline & Ic & $\mathrm{Ia}+\mathrm{IIa}$ & IIIa & \\
\hline 0.0 & 1.22 & 0 & 0 & 0.80 \\
\hline 0.43 & 0.89 & 0.51 & 0 & 0.92 \\
\hline 0.92 & 0.58 & 1.08 & 0 & 1.09 \\
\hline 1.34 & 0.24 & 1.14 & 0.44 & 1.19 \\
\hline 1.67 & $\leqslant 0.05$ & 1.10 & 0.84 & $\leqslant 1.31$ \\
\hline
\end{tabular}

a Summation diffusion current constant for waves Ic, Ia, IIa and IIIa.

an inflection in the $i-E$ profile and the obvious filming nature of single-drop $i-t$ profiles on Ia, which is not evident in the IIa process. Wave IIIa does not appear until the height of Ia plus IIa reaches its limiting value of $120 \mu \mathrm{A} \mathrm{cm}^{-2}$. At high anion concentration, Ila shows a slight maximum (Figs. 2 and 3 ).

\section{Controlled potential electrolysis}

Subtraction of background current, $i_{\mathrm{b}}$, from total current, $i_{\mathrm{t}}$, on CPE at $-2.42 \mathrm{~V}$ of $2.50 \mathrm{mM}$ thymine gave the current due to thymine reduction, $i$; the $\ln i$ vs. $t$ plot is linear only after $2400 \mathrm{~s}$ (Fig. 4). The $i-t$ data, planimeter inlegraled uver the first $2500 \mathrm{~s}$ and mathematically integrated from $2500 \mathrm{~s}$ to infinity, gave an $n$ of $0.73 \pm 0.02$.

Polarographic and voltammetric patterns for the thymine solution before and after c.p.e. are shown in Fig. 2. Because, peaks Ia to IIIa and IIc are not ob-

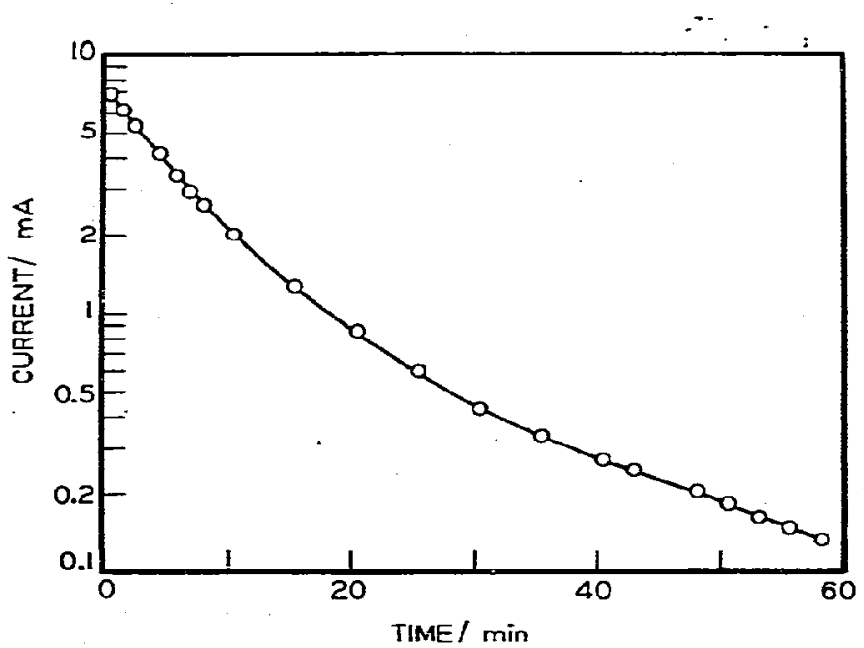

Fig. 4. Variation of the current with time during the controlled potential electrolysis of $2.50 \mathrm{mM}$ thymine. 
served before or after electrolysis of background alone, these are due to products of the thymine reduction. Addition of $\mathrm{HClO}_{4}$ to the electrolyzed solution decreased Ia and Ila, and caused Ic to reappear; addition of sufficient acid to eliminate Ia and IIa gave the Ic wave height expected for $1.67 \mathrm{mM}$ thymine. Addition of TEAH in a 1:1 ratio for $1.67 \mathrm{~m} M$ thymine produced polarographic and voltammetric results identical to those obtained after c.p.e.; $I_{\mathrm{d}}$ for the thymine anion is 1.29 .

The latter data indicate that two-thirds of the thymine is consumed by a non-faradaic process, e.g., deprotonation. Since the $n$ of 0.73 corresponds to slightly more than $2 e$ process for one-third of the thymine, all of the free radical formed on protonation of the initial $1 e$ reduction product is reduced in a second 1e process whose product reacts with thymine to generate thymine anion and dihydrothymine. Thus, the anion formed on free radical reduction is more basic than thymine itself.

The correspondence between cathodic and anodic $I_{\mathrm{d}}$ for both thymine and uracil confirms the $1 e$ nature for an $I_{\mathrm{d}}$ of 1.3. Due to the dependence of the e.c.e. mechanism on supply rate of proton donor, i.e., unreduced thymine, the larger $n$ obtained on c.p.e. compared to d.c. polarography at the same concentration is reasonable because of the convective stirring.

Although c.p.e. $n$ might be expected to reflect some weighted average of the d.c. poivircorraphic concentration-dependent $n$ (Fig. 1), i.e., the bulk concentration of reactants decirase as electrolysis proceeds, the $1.67 \mathrm{~m} M$ thymine anion in electrolyzed solution clearly indicates that the entire electrolysis proceeds via the expected high concentration mechanism.

\section{Product characterization}

It was not feasible to isolate individual dimetric and dihydrothymine products and to determine their structure, e.g., chromatography of electrolyzed solutions could not yield definitive results due to product instability and, even if successful, would not allow differentiation between anionic products and their protonated forms. Product recovery by freeze-drying was impractical due - to background electrolyte present and likelihood of alteration during evaporation. It was not possible to locate a solvent immiscible with DMSO, which would completely extract the reaction products without removing background electrolyte and from which the unaltered products could be recovered. Spectral identification of reduction products was only partially successful due to absence of reference spectra and instability of the likely products [9].

Consequently, the products have been characterized largely on the basis of faradaic patterns, behavior of solutions resulting from preparative electrolysis, well-characterized products obtained from related pyrimidines, reported products on radiolysis, and deductions based on electronic structure.

\section{Nature of mercury-thymidine film}

The mercury-thymine salt presumably involves $\mathrm{Hg}(\mathrm{I})$, as do the 2-HP and uracil anions $[1,2]$. The peak IIa shape suggests that the film deposited by Ia is not passivating in nature, i.e., Ila does not show a drawn-out leading edge 
(Figs. 2 and 3). The drawn-out nature of IIIa indicates that the film deposited by Ia plus IIa is passivating.

The amount of salt reduced in peak IIc, $Q_{c}$, is less than that deposited, $Q_{a}$, in peaks Ia, IIa plus IIIa. At $0.12 \mathrm{~V} \mathrm{~s}^{-1}, Q_{c} / Q_{\mathrm{a}}$ is 0.5 , independent of $Q_{a} / A$ magnitude $\left(150\right.$ to $\left.979 \mu \mathrm{C} \mathrm{cm}^{-2}\right)$; it is 0.7 at $0.36 \mathrm{~V} \mathrm{~s}^{-1}$, independent of $Q_{2} / A$

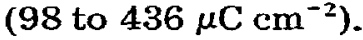

Three possibie magnitudes for the electrode area occupied by a thymine anion can be conceived, based on an anion diameter of $0.85 \mathrm{~nm}$, parallel to the ring plane and thickness of $0.4 \mathrm{~nm}$, assuming free methyl group rotation (CPK molecular models [10] were used to estimate molecular dimensions). For a flat orientation, the molecular swept area is $0.572 \mathrm{~nm}^{2}\left(2.90 \times 10^{-10} \mathrm{~mol} \mathrm{~cm}^{-2}\right.$; $28.0 \mu \mathrm{C} \mathrm{cm}^{-2}$ ); for a perpendicular orientation, the molecular area for close packing is $0.806 \mathrm{~nm}^{2}\left(5.4 \times 10^{-10} \mathrm{~mol} \mathrm{~cm}^{-2} ; 52 \mu \mathrm{C} \mathrm{cm}^{-2}\right)$ if $\mathrm{N}(1), \mathrm{N}(3)$ or $\mathrm{O}(4)$ lies flat on the surface, or $0.277 \mathrm{~nm}^{2}\left(6.0 \times 10^{-10} \mathrm{~mol} \mathrm{~cm} \mathrm{~cm}^{-2} ; 58 \mu \mathrm{C} \mathrm{cm}^{-2}\right)$ if $O(2)$ lies flat on the surface.

The probable reason for $Q_{c} / Q_{a}$ less than one is the number of layers of salt deposited by Ia to IIIa (Table 2); the minimum number of layers stripped by IIc at $1.67 \mathrm{mM}$ added TEAH is five. Such a large number of layers, which corresponds to a film thickness of $4-5 \mathrm{~nm}$ clearly suggests that small $Q_{\mathrm{c}} / Q_{\mathrm{a}}$ are caused by mcchanical film detachment due to non-adherence, whose extent would be expected to increase with both the amount of film deposited and the

\section{TABLE 2}

Number of layers of thymine anion deposited by cyclic peaks Ia, IIa and IIIa $\left(m_{a}\right)$ and stripped from the electrode surface by peak IIc $\left(m_{c}\right)$ as functions of scan-rate, amount of added base and assumed orientation ${ }^{a}$

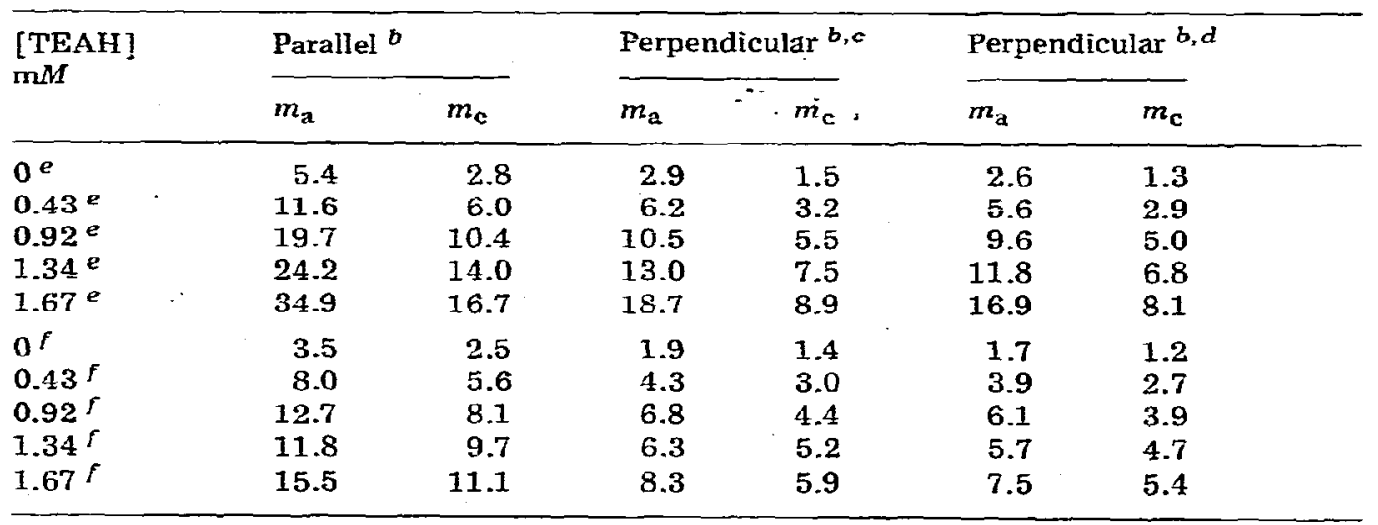

a Data are based on integrated area of cyclic voltammetric peaks for $1.67 \mathrm{mM}$ thymine (cf. Experimental section for method of peak area evaluation); assumed molecular dimensions for conversion from total charge under peaks to $m_{a}$ or $m_{c}$ are given in the discussion of mercury-thymine salt nature; electrode area was $0.0153 \mathrm{~cm}^{2}$.

$b$ Assumed orientation of plane of the thymine ring relative to the electrode surface.

$=\mathrm{N}(1), \mathrm{N}(3)$ or $\mathrm{O}(4)$ is assumed to be oriented toward the surface.

d $\mathbf{O}(2)$ is assumed to be oriented toward the surface.

Ccan-rate was $0.12 \mathrm{~V} \mathrm{~s}^{-1}$.

f Sean-rate was $0.35 \mathrm{~V} \mathrm{~s}^{-1}$. 
time from deposit to stripping; the observed results show both dependencies.

The large number of layers deposited and stripped indicates strong binding of the $\mathrm{Hg}(\mathrm{I})$-thymine salt to the $\mathrm{Hy}$ surface and strong cohesive forces between salt layers. Such strong cohesion was not observed for the Hg(I)-uracil salt [2]. The larger salt deposit attainable with thymine, which is indicative of lower monolayer passivation, may be due to the less closely packed pyrimidine rings for a perpendicular orientation (due to space required by the methyl groups), thereby facilitating diffusion of $\mathrm{Hg}(\mathrm{I})$ through the deposit to form additional layers. The larger fraction of salt stripped on reduction at fast $v$ indicates that the major source of film loss is mechanical detachment preceding or during film reduction. The shape of the film reduction peak on slow $v$ and its partial resolution into two peaks for high film deposits indicate a difference in ease of reduction between layers. At faster $v$ and high anion $c$, only one peak appears at a potential corresponding to the more difficultly reducible layers; hence, the layers closest to the electrode surface seem to be the most difficult to reduce.

\section{MECHANISM}

Several aspects of the behavior expected for the mechanism, which best fits the information available (Fig. 5), can be specified. Reduction of $\dot{\mathrm{R}} \mathrm{H}_{2}$ is generally easier than that of RH. Based on sludies of similar compounds $[2,11], k_{3}$

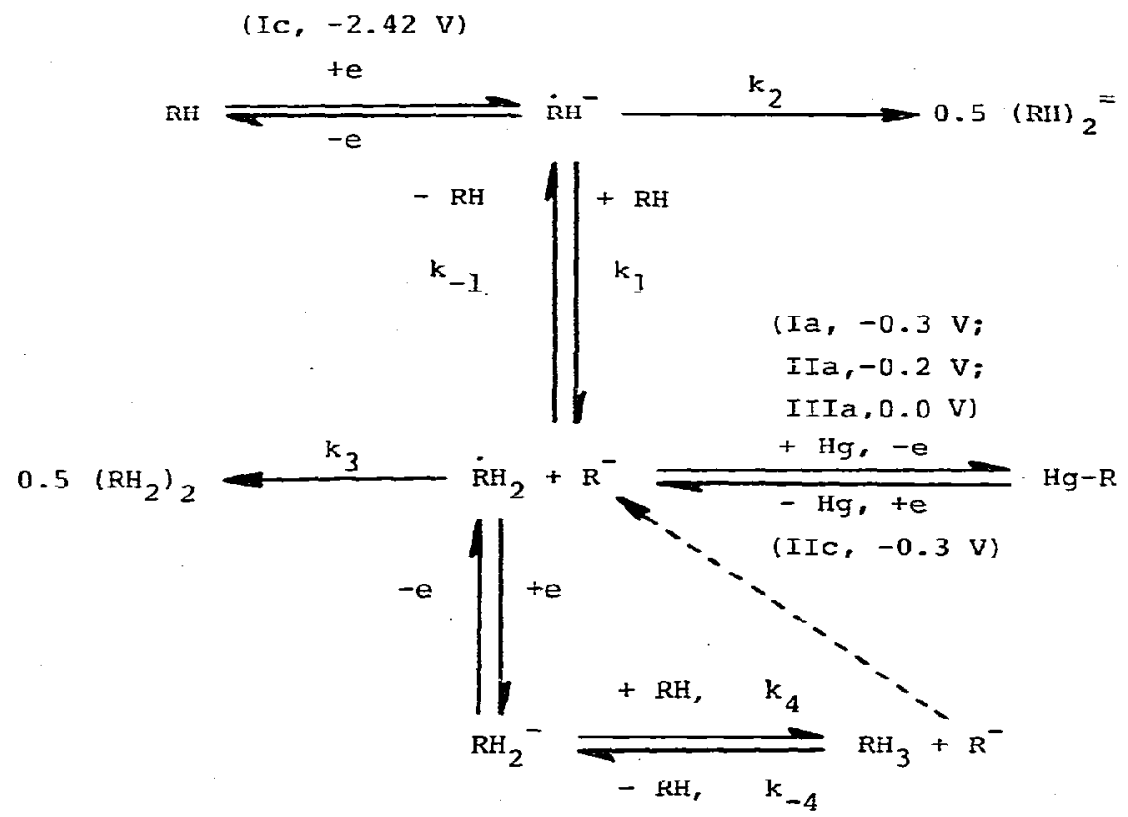

Fig. 5. Reduction mechanism for thymine in DMSO. Symbols: $\mathbf{R H}$, thymine; $\mathbf{R H}^{-}$, radical anion, $\hat{\mathrm{H}}_{2}$, free radical; $\mathbf{R H}_{2}^{-}$, reduced free radical; $\mathrm{RH}_{3}$, dihydrothymine; $\mathrm{R}^{-}$, thy mine anion. The $k$ represent rate constants; the Roman numbers are wave and peak designations (cf. Fig. 3). Potentials are $E_{\mathrm{p}}$ for $1.67 \mathrm{~m} M$ thymine; for Ia, IIa, IIIa and IIc, solution is also $1.67 \mathrm{~m} M$ in TEAH. 
for $\mathrm{RH}_{2}$ dimerization may be $10^{6}$ to $10^{8} \mathrm{M}^{-1} \mathrm{~s}^{-1}$; because of electrostatic repulsion, $\dot{\mathrm{R}} \mathrm{H}^{-}$dimerization should be slower, e.g., $k_{2}$ of $10^{4}$ to $10^{6} \mathrm{M}^{-1} \mathrm{~s}^{-1}$. Because the protonation does not involve reaction between similarly charged molecules with concomitant repulsion and radical anions are very basic, protonations of $\dot{\mathbf{R}} \mathrm{H}^{-}$should be more rapid than its dimerization, i.e., $k_{1}$ is greater than $k_{2}$; however, since the protonation does not involve two molecules with the strong reaction tendency of free radicals, e.g., dimerization where charge repulsion is not involved, the protonation is expected to be slower than dimerization of R $\mathrm{H}_{2}$, i.e., $k_{1}$ less than $k_{3}$.

These kinetic relations may not be valid for thymine in respect to the $\dot{\mathrm{R}} \mathrm{H}_{2}$ dimerization; at high $c$ where dimerization should be favored, all of the initially reduced thymine undergoes a second $1 e$ transfer. This presence of an e.c.e. process, which is not observed for uracil [2], suggests that $\dot{\mathrm{R}} \mathrm{H}_{2}$ dimerization for thymine is slower than that of uracil; this is reasonable, if dimerization occurs at $C(4), C(5)$ or $C(6)$, since the 5-methyl group on thymine might sterically hinder dimerization.

The thymine anion, as expected, is much more difficultly reducible than thymine itself; its reduction is not observed within the available potential range.

\section{Calculuted and experimental ease of reduction}

Fublished calculations of molecular orbital energy levels are fewer for thymine than for uracil (cf. ref. 2 and references cited therein.) The calculated $0.03 \mathrm{~V}$ difference in electron affinities between uracil [12] and thymine [13] is in good agreement with the difference in $E_{1 / 2}(0.07 \mathrm{~V}$ at $0.2 \mathrm{mM}$ to $0.05 \mathrm{~V}$ above $1 \mathrm{mM}$ ); the small difference is within the uncertainty of the calculated electron affinities. Thus, thymine reduction appears to be kinetically hindered, similarly to uracil [2], due to a combination of heterogeneous and homogeneous kinetic effects. This is supported by the agreement between $i_{p} / A c v^{1 / 2}$ at $0.35 \mathrm{~V} \mathrm{~s}^{-x}$ and $I_{\mathrm{d}}$, assuming an $\alpha$ of 0.5. The latter, which is larger than the $\alpha$ of $\mathbf{G . 3 9}$ observed for uracil [2], may be due to involvement of the second step.

\section{Sites of electron entrance and residence}

Since rapid electron rearrangement may occur after initial addition to pyrimidine [14], the structure of detectable intermediates and final products is not conclusive regarding the site of electron injection.

The probable site of the added electron on uracil reduction in DMSO is C(4), once the generated anion is protonated [2]. The radical anion in basic aqueous media is predicted to be the 3-H lactim with the unpaired electron delocalized [15]. The electron first added to thymine likely also resides at $C(4)$ after protonation. The electron density, bond order and free valence in the thymine $C(5)=C(6)$ region are high, in agreement with observed chemical oxidation at $5,6 \mathrm{C}=\mathrm{C}[13]$; the latter, consequently, is unlikely to be the site of initial reduction, in as much as the most easily oxidized site is rarely also the most easily reduced site.

The strongest argument for initial $1 e$ reduction at $C(4)$ rather that at the 
other $\mathrm{C}=\mathrm{O}$ site, $\mathrm{C}(2)$, is the fact that the thymine free radical is reduced while the uracil free radical dimerizes before it can be reduced [2]. The considerably slower thymine free radical dimerization suggests that the 5-methyl group might influence the dimerization rate through steric hindrance, a likely effect only if the dimerization site were $C(4)$. Reduction at $C(4)$ is also supported by Hayon's observation that on pulse radiolysis of thymine the ketyl radical at $C(4)$ is formed [9]. Thus, the free radical formed on initial $1 e$ reduction appears to be species I. The second electron likely also goes onto $\mathrm{C}(4)$, followed by proton abstraction to form species II.<smiles></smiles>

(I)<smiles>CC1=CNC(=O)NC1O</smiles>

(ㅁ)

Concentration and kinetic dependence of reduction process

The high-concentration $n$ of 0.59 seen on d.c. polarography indicates that species I is not quantitatively reduced to II. This lower yield of II, as compared to c.p.e., is likely due to the small unreduced thymine concentration near the electrode surface on polarography and to the relative acidity of I and thymine. The free radical $\mathrm{pK}$ a $7.2 \pm 0.3$ [9] makes $I$ more acidic by ca. two $\mathrm{pK}$ units than thymine. Thus, although the electrochemical results clearly indicate that thymine is chemically consumed to yield the conjugate base, from which formation of the free radical can be inferred, the protonation reaction must be explained by kinetic, rather than equilibrium, arguments.

Any reaction consuming the free radical, e.g., dimerization or reduction, should shift the proton transfer equilibrium to favor free radical formation. At sufficiently low proton donor (thymine) concentration near the electrode surface, e.g., d.c. polarography on the $i_{1}$ plateau, the radical anion must diffuse from the electrode into a region of higher thymine concentration to acquire a proton. Under such circumstances, appresiable dimerization of free radical occurs before it can diffuse back to the electrode and be reduced. At high thymine concentration near the electrode, e.g., c.p.e. with convective stirring, the radical anion will be protonated near the electrode surface and the free radical will be reduced more rapidly than it is consumed by the sterically hindered dimerization.

The high-concentration mechanistic process differs from that of uracil [2] principally in terms: of free radical dimerization rate. The $p K_{a}$ of $7.3 \pm 0.3$ for the uracil free radical [9] indicates that kinetic, rather than equilibrium, arguments must also explain radical anion protonation by unreduced uracil. However, the uracil free radical dimerization is not sterically hindered and is extremely rapid; thus, even on c.p.e., the free radical dimerizes before it can be reduced.

The low-concentration results, i.e., $n$ approaching one, agree with the u.v. 
spectral behavior of thymine on adding TEAH. The inability of TEAH at a $3: 1$ ratio to deprotonate $0.047 \mathrm{mM}$ thymine indicates that the thymine to radical anion proton-transfer reaction is unlikely at low thymine concentration. The $0.35 \mathrm{~m} M$ TEAH concentration necessary to convert all thymine to its conjugate base at this thymine level is only slightly below the thymine concentration at which the $I_{d}-c$ relation plateaus, suggesting that TEAH and the thymine radical anion have equivalent basic strength in DMSO, possibly due to ion-pairing of $\mathrm{OH}^{-}$. The possibility that a larger than $1: 1 \mathrm{TEAH}$ : thymine ratio is generally required for complete thymine conversion to its conjugate base is precluded by behavior at $1.67 \mathrm{~m} M$ thymine, for which wave Ic disappears at $1: 1$ added TEAH.

At low concentration, e.g-, below $0.4 \mathrm{~m} M$, the biomolecular protonation reaction is slowed under d.c. polarographic conditions to the point that the radical anion must diffuse far from the electrode before the protonation rate becomes appreciable. Although the fate of the radical anion under these conditions is unclear, dimerization seems more likely than protonation followed by dimerization of the resulting free radical. Since the $\mathrm{p} K_{\mathrm{a}}$ of thymine and its free radical indicate that protonation is not favored, and since free radical formation far from the electrode results in a low free radical concentration, its dimerization will be slow; without the driving force of a rapid, irreversible following reaction, the proton transfer reaction will not proceed appreciably, and the ultimate fate of the radical anion will be dimerization. Thus, very little thymine is consumed by the protonation reaction and the observed $n$ approaches one.

\section{ACKNOWLEDGMENTS}

The authors thank the National Science Foundation and the Horace $\mathrm{H}$. Rackham School of Graduate Studies of The University of Michigan, which helped support the work described, and Mr. Mark Hyman, who assisted with the experimental work.

\section{REFERENCES}

1 T. Wasa and P.J. Elving, J. Electroanal. Chem., 91 (1978) 249.

2 T.E. Cummings and P.J. Elving, J. Electroanal. Chem., 94 (1978) 123.

3 J.S. Kwiatkowski and B. Pullman in A.R. Katritzky and A.J. Boulton (Eds.), Advances in Feterocyclic Chemistry, Vol. 18, Academic Press, New York, 1975, pp. 199-335.

4 P.J. Elving, Can. J. Chem.. 55 (1977) 3392.

5 D.A. Fall and P.J. Elving, Anal. Chim. Acta, 39 (1967) 141.

6 T.E. Cunmines, M.A. Jensen and P.J. Elving, Electrochim. Acta, $23(1978) 1173$.

7 T.E. Cummings and P.J. Elving. Anal. Chem.. 50 (1978) 480.

8 H. Matsuda, Bull. Chem. Soc. Jap., 26 (1953) 342.

9 E. Hayon. J. Chem. Phys.. 51 (1969) 4881.

10 CPK Precision Molecular Models. The Ealing Corpotation, 2225 Massachusetts Ave., Cambridge. Mass. 02140

11 P.J. Elving in G. Milazzo (Exi.), Topics in Bioelectrochemistry and Bioenergetics, Vol. I, John Wiley, London, 1976, pp. 179-286.

12 H. Berthod, C. Giessner-Prettre and A. Pulman, Theor. Chim. Acta, 5 (1966) 53.

13 A. Denis and A. Pullman, Theor. Chim. Acta. 7 (1967) 110.

14 J.E. O'Reilly and P.J. Elvine, J. Amer. Chem. Soe., 93 (1977) 1871 .

15 A. Grimison and M.K. Eberhardt. J. Phys. Chem., 77 (1973) 1673. 